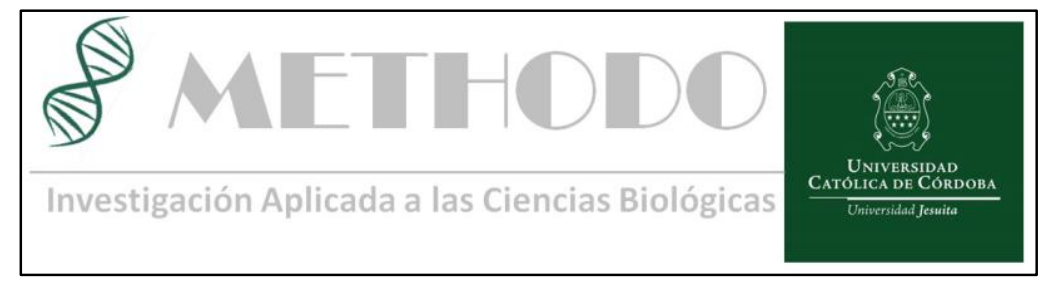

ARTICULO ORIGINAL Methodo 2017;2(3): 79-89

DOI: $10.22529 / \mathrm{me} .2017 .2(3) 02$

Recibido 19 Jun 2017 | Aceptado 30 Jul 2017 | Publicado 30 Sep 2017

\title{
Identificación de población de riesgo para alergia a la leche de vaca
}

\section{Identification of population at risk for allergy to cow's milk}

\author{
Boudet RV¹, Copioli $\mathrm{JC}^{2}$, Muiño $\mathrm{JC}^{3}$, Geréz de Burgos N${ }^{1}$, Chaig MR ${ }^{1}$, Damilano ${ }^{4}$, Chaig R.
}

\section{Resumen}

ANTECEDENTES: Los genotipos asociados con la alergia a la leche de vaca (ALV) son desconocidos. Aún no han podido ser replicados en poblaciones independientes, y podrían ser responsables de la marcada variabilidad de la respuesta clínica individual a las proteínas lácteas.

OBJETIVO: Caracterizar haplogrupos, de la Región D-Loop del ADN mitocondrial, en un grupo de niños ALV, con el fin de arribar a un mejor conocimiento de la herencia biológica y genética en la etiología de la enfermedad.

POBLACION Y METODO: Diseño: Análisis de mutaciones o variantes de la región D-loop del genoma mitocondrial. Población: 41 niños de ambos sexos de 0-2 años, 11 alérgicos ALV y 30 controles. (Río Cuarto, Córdoba, Argentina) Los pacientes ALV se dividieron, según la sintomatología que presentaban en 6 casos con Dermatitis Atópica (DA) + Enfermedad Gastrointestinal (EGI) y en 5 casos con Rinitis y Asma (RA).

La Región D-Loop del genoma mitocondrial se amplificó por PCR. El análisis filogenético fue calculado usando el programa CLUSTAL OMEGA, the Neighbor-Joining, BLOSUM62, con los datos estudiados y grabados por Jukes-Cantor y luego con Kimura-2, programas específicos disponibles (software).

RESULTADOS: Se encontró una mutación o variante nucleotídica no descripta T16519C en la transición de haplogrupos asociada a pacientes ALV con DA+EGI en 6/6 casos, comparados con $5 / 5$ casos con RA que no la presentaron, mientras que en los controles se la observó solo en 6/30, p=0,0312; RR 2,900.

CONCLUSIONES: Estos hallazgos sugieren que esta mutación probablemente aumente la posibilidad de padecer ALV asociada con DA+EGI.

Palabras Claves: Alergia a la leche de vaca, haplogrupos, niños, mutación no descripta, genoma mitocondrial

\section{Abstract}

BACKGROUND: Genotypes associated to cow's milk allergy (CMA) are unknown. They have not been replicated in independent populations, and could be responsible for the marked variability in individual clinical response to milk proteins.

OBJECTIVE: To characterize haplogroups of the D-Loop region of mitochondrial DNA in a group of children allergic to cow's milk in order to arrive at a better understanding of biological and genetic heritability in the etiology of the disease.

POPULATION AND METHOD: Design: Analysis of mutations or variants of the D-loop of mitochondrial genome region. Population: 41 children of both sexes from 0-2 years, 11 with CMA and 30 healthy subjects (controls). (Río Cuarto, Córdoba, Argentina). The CMA patients were divided according to the symptoms presenting in: 6 cases with Atopic Dermatitis (AD) +

Revista Methodo: Investigación Aplicada a las Ciencias Biológicas. Facultad de Medicina. Universidad Católica de Córdoba. Jacinto Ríos 571 Bo Gral. Paz. X5004FXS. Córdoba. Argentina. Tel.: (54) 351 4517299 / Correo: methodo@ucc.edu.ar / Web: methodo.ucc.edu.ar | ARTICULO ORIGINAL Methodo 2017;2(3): 79-89 
Boudet RV, Copioli JC, Muiño JC, Geréz de Burgos N, Chaig MR, Damilano G, Chaig R. Identificación de población de riesgo para alergia a la leche de vaca

Gastrointestinal disease (GID) and in 5 cases with Rhinitis and Asthma (RA). The D-Loop Region of mitochondrial genome was amplified by PCR. Phylogenetic analysis was calculated using the program CLUSTAL OMEGA, the Neighbor-Joining, BLOSUM62, with studied and recorded by Jukes-Cantor data and then with Kimura-2, available specific programs (software). RESULTS: We found a non-descript mutation or variant nucleotide T16519C in the transition of haplogroups associated with CMA patients with AD+ GID in 6/6 cases, compared with 5/5 cases with RA that failed it, whereas in controls was observed it only in $6 / 30, p=0,0312 \mathrm{RR}$ 2,900 .

CONCLUSIONS: These features suggest that this mutation probably increases the possibility of suffering CMA associated with $A D+$ GID.

Key words: Cow's Milk Allergy, haplogroups, children, not decipher mutation, mitochondrial genome..

1. Cátedra de Bioquímica y Biología Molecular, Universidad Nacional de Córdoba (UNC) 2. Cátedra de Unidad Hospitalaria de Medicina interna № 1 Hospital Córdoba Facultad de Ciencias Médicas (FCM), UNC 3. Cátedra de Unidad Hospitalaria de Medicina interna № 4 Hospital Misericordia, FCM, UNC, 4. Facultad de Ciencias Humanas, Universidad Nacional de Río Cuarto. 5. Ex Profesor Titular de La Cátedra de Operaciones 1- en la Carrera de Ingeniería Química - Universidad Tecnológica de Córdoba.

Correspondencia: Raúl Vicente Boudet, Domicilio: Mendoza 844 (5800) Río Cuarto, e-mail: rulovboudet@yahoo.com.ar

\section{INTRODUCCIÓN}

La alergia a los alimentos es un problema de salud pública a nivel global. Su incidencia ha aumentado en la última década más rápidamente de lo que permitirían los cambios en el genoma. Sin embargo, todavía no se ha podido determinar cuáles son los factores ambientales que podrían interactuar con el riesgo adquirido genéticamente para desarrollar la enfermedad (1, 2). Algunos individuos poseen factores genéticos que confieren susceptibilidad o resistencia a una determinada patología en un entorno particular.

La historia familiar es un potente factor de riesgo para el desarrollo de la alergia alimentaria. Hay buena evidencia proveniente de estudios en gemelos, que señalan la importancia de la variación genética, más aún cuando se asocia con otras enfermedades atópicas $(3,4,5)$. La predisposición genética conduce a la producción de citocinas predominantemente TH2 (IL4, IL-5, IL-9 y IL-13) que contribuyen a la sensibilización alérgica, originada en defectos intrínsecos de células T o de células presentadoras de antígenos (6). La alergia a la leche de vaca (ALV) es la principal causa de alergia alimentaria, afecta mayormente a los niños y no existe suficiente información referente a tendencias geográficas en su desarrollo. En ese sentido, los genotipos asociados con la ALV son desconocidos (aún no han podido ser replicados en poblaciones independientes) y podrían ser responsables de la marcada variabilidad de la respuesta clínica

Revista Methodo: Investigación Aplicada a las Ciencias Biológicas. Facultad de Medicina. Universidad Católica de Córdoba. Jacinto Ríos 571 Bo Gral. Paz. X5004FXS. Córdoba. Argentina. Tel.: (54) 351 4517299 / Correo: methodo@ucc.edu.ar / Web: methodo.ucc.edu.ar | DOI: 10.22529/me.2017.2(3)02 
Boudet RV, Copioli JC, Muiño JC, Geréz de Burgos N, Chaig MR, Damilano G, Chaig R. Identificación de población de riesgo para alergia a la leche de vaca

individual a las proteínas lácteas (7).

Por eso, en la actualidad el riesgo de padecer ALV es definido de acuerdo a los antecedentes familiares $(8,9)$. La población humana ha acumulado un alto número de substituciones de bases en el ADN mitocondrial (ADNmt) a lo largo de linajes maternales, en los cuales las combinaciones específicas constituyen los haplogrupos mitocondriales supuestos $(10,11)$. Los haplogrupos ADNmt específicos para una población, pueden ser funcionalmente diferentes y ejercer influencias que podrían afectar los resultados de las enfermedades, ya sea exacerbando, retrasando o bien aminorando la sintomatología dada (12, 13, 14).

El avance en los estudios de secuenciación de la Región Hipervariable uno $(\mathrm{HVI})$ de la región D-Loop en el ADNmt ha revelado que la tasa de mutación es diferente a lo largo del segmento. Algunas posiciones de la Región $\mathrm{HVI}$, son extremamente variables entre los linajes, mientras que otras, relativamente constantes (15). El objetivo de esta investigación fue caracterizar haplogrupos de la Región D-Loop del ADN mitocondrial, en un grupo de niños alérgicos a la leche de vaca, con el fin de arribar a un mejor conocimiento de la herencia biológica y genética en la etiología de la enfermedad.

\section{POBLACION Y MÉTODOS}

Se estudiaron, 41 niños de 0 a 2 años de edad y de ambos sexos, que viven en la ciudad de Río Cuarto, Córdoba, Argentina. Once (11) de ellos tenían diagnóstico de ALV y treinta (30) individuos sanos conformaron el grupo control. En todos los pacientes el diagnóstico se confirmó, luego de dos semanas de exclusión del alimento, por medio del desafío oral abierto, atendiendo a razones prácticas como son la corta edad de los niños y la baja sensibilidad de las pruebas diagnósticas realizadas (RAST IgE específica y Prick Test con extractos estandarizados).

Los niños ALV se dividieron de acuerdo al tipo de manifestaciones clínicas con que iniciaron la enfermedad en 6 casos con Dermatitis Atópica (DA) +

Enfermedad Gastrointestinal (EGI) y en 5 casos con Rinitis y Asma (RA).

La región D-Loop y sus segmentos o Regiones Hipervariables HVI, HVII y HVIII del Genoma mitocondrial (Gmt), fue amplificada por Reacción en Cadena de la Polimerasa (PCR). Los "primers" utilizados fueron: Fw 16033 a 16055 y Rev. 50 a 29 (RHVI); Fw 29 a 51 y Rev. 713 a 693 (HVII y III). Las condiciones de la mezcla de incubación, para amplificar los productos en la PCR, tenían una temperatura de annelling (para los diferentes segmentos) en un rango de 
Boudet RV, Copioli JC, Muiño JC, Geréz de Burgos N, Chaig MR, Damilano G, Chaig R. Identificación de población de riesgo para alergia a la leche de vaca

$56^{\circ} \mathrm{C}$ a $58^{\circ} \mathrm{C}$. Posteriormente se realizó

electroforesis en geles de agarosa al

$2 \%$, para la visualización de las bandas de ADN, con luz UV. Cada fragmento fue purificado y mandado a secuenciar para su análisis (dos determinaciones). Los resultados fueron comparados con la puesta del Consenso de la secuencia de Cambridge (GenBank Accession No.: NC_012920.1). Además, se utilizó la base de datos de GenBank (http://www.ncbi.nlm.nih.gov/ MITOMAP), y las base de datos The Human Mitochondrial Genome Database H.M.G.D. (www.mitomap.org; www.genpat.uu.se/mtDB/).

\section{Análisis Estadístico}

Para determinar la asociación entre los haplogrupos y la enfermedad, se utilizaron tablas de contingencia y cálculo de riesgo (OR). El resumen estadístico de la diversidad genética fue calculado usando el programa CLUSTAL OMEGA versión 2.0.12 (Múltiples Alineaciones de la Secuencia). Para la filogenia se utilizó el Neighbor-Joining (NJ), BLOSUM62 (BLOcks of Amino Acid SUbstitution

\section{RESULT A DOS}

La Región D-Loop del Gmt fue comparada con cada matriz de la Región Hipervariable (HVI, HVII y HVIII) de cada paciente (paso 1). Se observó que las variantes de la Región HVI, se distribuyen acercándose o
Matrix, o matriz de sustitución de bloques de aminoácidos) con datos estudiados y registrados por JukesCantor y luego con Kimura-2 (16, 17, 18, 19).

Finalmente, se generaron nuevos árboles filogenéticos utilizando los métodos de Average distance tree using PID; Neighbour Joining tree using PID, Average distance tree using BLOSUM62, con el fin de compararlos con los obtenidos.

\section{Consideraciones éticas}

Este estudio se realizó previo consentimiento informado y se con la normativa de la Declaración de Helsinki, Buenas Prácticas Clínicas de ANMAT y Ley Provincial № 9694. E estudio fue aprobado Comité de Bioética del Ministerio de Salud, Gobierno de la Provincia de Córdoba. Resolución № 296.

Se asegura protección de datos personales de los pacientes según la Ley 25.326. Los autores declaran no tener conflictos de intereses.

alejándose del Gmt. El árbol filogenético usado para guía del proceso de múltiple alineamiento final, se calculó de la distancia matriz del paso 1, usando Neighbour Joining method. Este árbol indica qué 
Boudet RV, Copioli JC, Muiño JC, Geréz de Burgos N, Chaig MR, Damilano G, Chaig R. Identificación de población de riesgo para alergia a la leche de vaca

pacientes o controles se alejan más del

ADNmt. El paciente 13a BS, fue, en

nuestra investigación, el más próximo

al Gmt, el 2a CG el más alejado, y el 1a

MJC está ancestrado con el Gmt y con

13a BS, pero se aleja ancestralmente

de los otros. El árbol dibujado para la

HVII y HVIII, no refleja lo mismo,

aunque si lo hace para 2b CG y $17 b$

LB. Esto demuestra los cambios

sufridos en el tiempo para ciertas

variables (cambios nucleotídicos).

En la Tabla 1, se muestran los

haplogrupos y la identificación de cada

variante o mutación de la población

estudiada, en las Regiones HVI, HVII y

HVIII, siguiendo los datos registrados

en el mapa genético

(www.mitomap.org;

www.genpat.uu.se/mtDB).

Tanto los niños ALV como los

controles, presentaron prevalencia de

los siguientes haplogrupos:

RXIZGDZV; $A+C+B$; TJgroup;

UK,HVgroup; q; z; v; A+C1B y ACB, (el signo+ indica frecuencia). En la Tabla

2 , se señala su distribución porcentual

por áreas geográficas. Además, se

identificó cada variante, su frecuencia,

su pertenencia a un haplogrupo o no, si

es una variable identificada en el

(www.mitomap.org;

www.genpat.uu.se/mtDB/), con que

otras variantes se encuentra asociada y

de qué región ancestral proviene. Este

procedimiento permitió observar la

\begin{tabular}{l|c|}
\hline \multicolumn{2}{|l|}{ Tabla 1. Haplogrupos y variantes genéticas en } \\
los niños estudiados \\
\hline HAPLOGRUPOS & n \\
\hline $\begin{array}{l}\text { WT, A+C B } \\
\text { (Europa, Asia) }\end{array}$ & 2 \\
$\begin{array}{l}\text { RXIZGDZV, A+C+B } \\
\text { (Europa, Asia) }\end{array}$ & 10 \\
ACBV \\
(Asia, Europa) \\
$\begin{array}{l}\text { WDEG, ACB } \\
\text { (Europa, Asia) }\end{array}$ & 1 \\
$\begin{array}{l}\text { RXIZGD, UK,Hvgroup; Q, A+C1+B } \\
\text { (Europa, Asia) }\end{array}$ & 5 \\
$\begin{array}{l}\text { B++ AC1 } \\
\text { (Asia) }\end{array}$ & 5 \\
$\begin{array}{l}\text { UK, Hvgroup, Q, Z, V, A+C1B } \\
\text { (Europa, Asia) }\end{array}$ & 1 \\
T, J, Tjgroup, WT, A+ C+ \\
(Europa, Asia) \\
$\begin{array}{l}\text { Q } \\
\text { (Asia) }\end{array}$ & 6 \\
$\begin{array}{l}\text { T, J, Tjgroup, UK, Hvgroup, } \\
\text { Q, DE, A++C+B } \\
\text { (Europa, Asia) } \\
\text { ACB } \\
\text { (Asia) }\end{array}$ & 6 \\
Total & 1 \\
\hline
\end{tabular}

prevalencia del haplogrupo C; TJ

group; y de la mutación o variante no

descripta T16519C (Figura 1).

El diagnóstico de ALV y la presencia de

la variante nucleotídica no descripta

T16519C se asociaron

significativamente al 10\% ( $p=0,095)$;

Coeficiente de contingencia $=0,252$

(Prueba chi-cuadrado para la

asociación entre grupo de pertenencia

y presencia de la mutación). Un

paciente que posea dicha mutación, tiene mayor probabilidad de ser ALV que aquel que no la presenta:

OR=3,300, IC95\% 0,785; 13,879 (Tabla

3).

Revista Methodo: Investigación Aplicada a las Ciencias Biológicas. Facultad de Medicina. Universidad 
Boudet RV, Copioli JC, Muiño JC, Geréz de Burgos N, Chaig MR, Damilano G, Chaig R. Identificación de población de riesgo para alergia a la leche de vaca

La evaluación del indicador pudo

resumirse como: Área ROC global

64\%; Sensibilidad 55\%; Especificidad

73\%; Predictivo Positivo 43\%;

Predictivo Negativo $82 \%$.

Se halló a la variante T16519C no

descripta en la transición de

haplogrupos asociada con los

pacientes ALV que iniciaron la

enfermedad con manifestaciones

clínicas de Dermatitis Atópica (DA) +

Enfermedad Gastrointestinal (EGI) en
6/6 pacientes, mientras que no hubo asociación en $5 / 5$ pacientes que presentaron manifestaciones clínicas de inicio de Rinitis y Asma (RA), $\mathrm{p}=0.0001$.

En el grupo control solo 6/30 individuos poseían la variante $\mathrm{T} 16519 \mathrm{C}$ no descripta con una asociación también significativa, $p=0,0312 R R 2,900$

(Figura 2).

Tabla 2. Distribución de haplogrupos por regiones geográficas en el grupo de niños alérgicos a la leche de vaca y en los controles

\begin{tabular}{|l|c|c|c|c|c|}
\hline HAPLOGRUPO & $\begin{array}{c}\text { Controles } \\
(\mathrm{n})\end{array}$ & $\begin{array}{c}\text { ALV } \\
(\mathrm{n})\end{array}$ & $\begin{array}{c}\text { Controles } \\
(\%)\end{array}$ & $\begin{array}{c}\text { ALV } \\
(\%)\end{array}$ & $\begin{array}{c}\text { Total } \\
(\%)\end{array}$ \\
\hline Europeo & 21 & 9 & 51,22 & 21,95 & 73,17 \\
\hline Asiático - Europeo & 5 & 0 & 12,20 & 0,00 & 12,20 \\
\hline Asiático - Americano & 3 & 2 & 7,32 & 4,88 & 12,20 \\
\hline Africano & 1 & 0 & 2,44 & 0 & 2,44 \\
\hline Sub-Total & 30 & 11 & 73,17 & 26,83 & 100,00 \\
\hline
\end{tabular}

$\mathbf{A L V}=$ grupo conformado por niños alérgicos a la leche de vaca

Controles=grupo de niños sanos 
Boudet RV, Copioli JC, Muiño JC, Geréz de Burgos N, Chaig MR, Damilano G, Chaig R. Identificación de población de riesgo para alergia a la leche de vaca

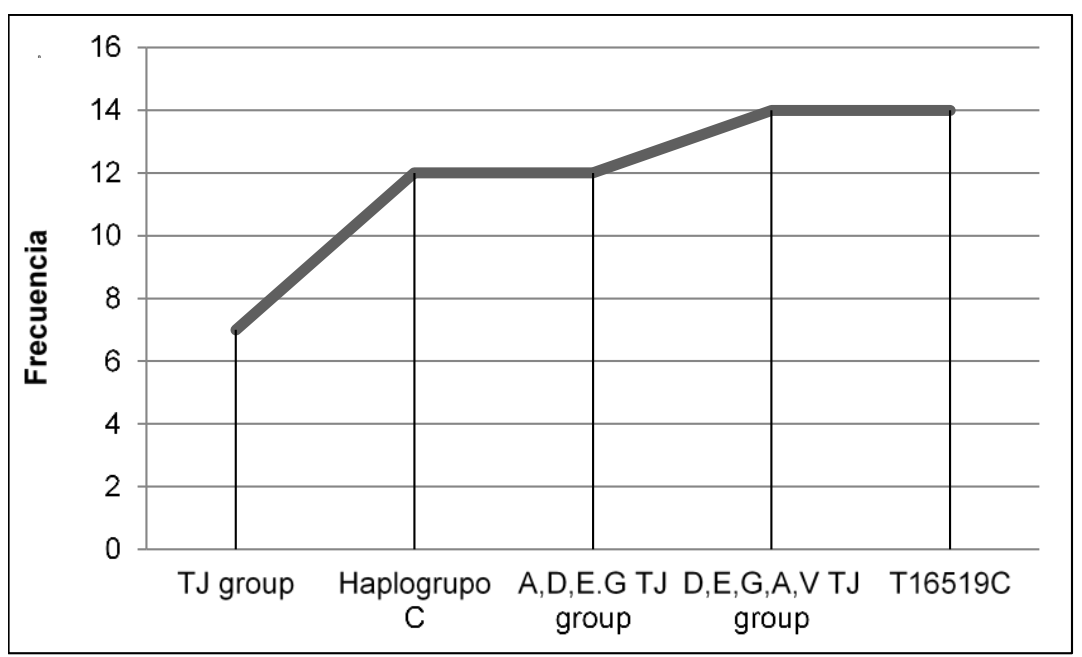

Figura 1. Prevalencia de haplogrupos y de la variante no descripta T16519C en los niños estudiados

Tabla 3. Presencia de la variante no descripta (T16519C) en niños alérgicos a la leche de vaca vs. controles

\begin{tabular}{|c|c|c|c|c|c|}
\hline \multirow{2}{*}{\multicolumn{3}{|c|}{ Variante no descripta (T16519) }} & \multicolumn{2}{|c|}{ Grupo } & \multirow[b]{2}{*}{ Total } \\
\hline & & & ALV & Controles & \\
\hline \multirow{4}{*}{\multicolumn{2}{|c|}{ NO }} & $\mathrm{n}$ & 5 & 22 & 27 \\
\hline & & $\%$ dentro de Mutación & $18,5 \%$ & $81,5 \%$ & $100,0 \%$ \\
\hline & & $\%$ dentro de Grupo & $45,5 \%$ & $73,3 \%$ & $65,9 \%$ \\
\hline & & $\%$ del total & $12,2 \%$ & $53,7 \%$ & $65,9 \%$ \\
\hline \multirow{4}{*}{\multicolumn{2}{|c|}{ SI }} & $\mathrm{n}$ & 6 & 8 & 14 \\
\hline & & \% dentro de Mutación & $42,9 \%$ & $57,1 \%$ & $100,0 \%$ \\
\hline & & $\%$ dentro de Grupo & $54,5 \%$ & $26,7 \%$ & $34,1 \%$ \\
\hline & & $\%$ del total & $14,6 \%$ & $19,5 \%$ & $34,1 \%$ \\
\hline \multirow{4}{*}{\multicolumn{2}{|c|}{ Total }} & Recuento (n) & 11 & 30 & 41 \\
\hline & & \% dentro de Mutación & $26,8 \%$ & $73,2 \%$ & $100,0 \%$ \\
\hline & & $\%$ dentro de Grupo & $100,0 \%$ & $100,0 \%$ & $100,0 \%$ \\
\hline & & $\%$ del total & $26,8 \%$ & $73,2 \%$ & $100,0 \%$ \\
\hline
\end{tabular}

$\mathbf{A L V}=$ grupo conformado por niños alérgicos a la leche de vaca

Controles=grupo de niños sanos 
Boudet RV, Copioli JC, Muiño JC, Geréz de Burgos N, Chaig MR, Damilano G, Chaig R. Identificación de población de riesgo para alergia a la leche de vaca

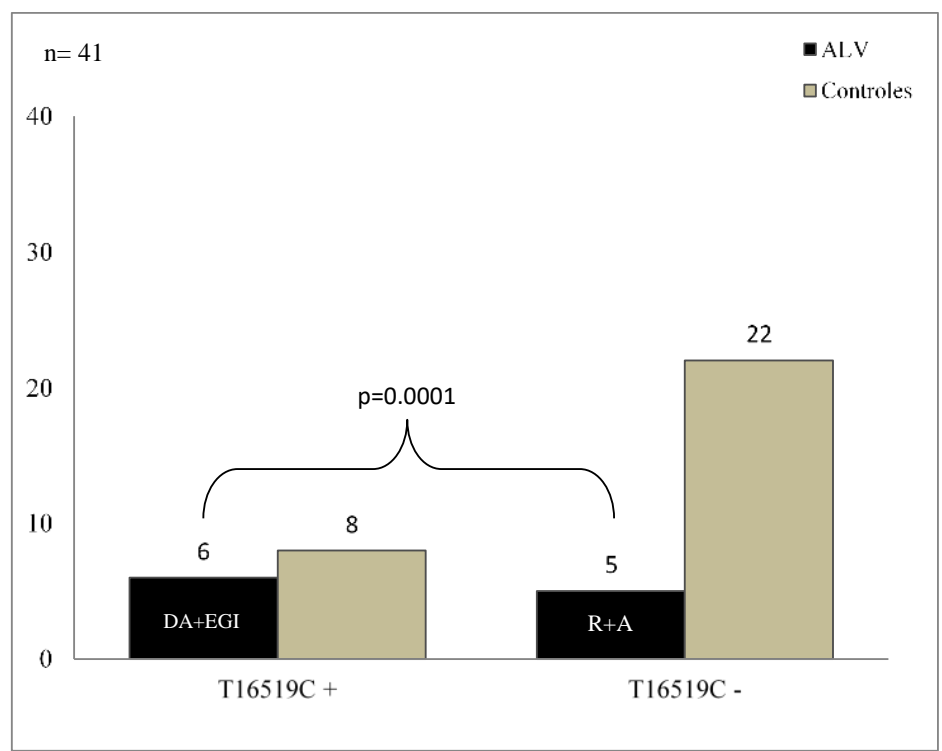

Figura 2. Relación entre manifestaciones clínicas y la presencia de la variante no descripta T16519C en niños alérgicos a la leche de vaca vs.controles $A L V=$ grupo de niños alérgicos a la leche de vaca; Controles: pacientes sanos $\mathrm{DA} / \mathrm{EGl}=$ Dermatitis Atópica/Enfermedad Gastrointestinal

$R / A=$ Rinitis $/$ Asma

\section{DISCUSIÓN}

En los últimos años se han descrito mutaciones que se han asociado con síndromes clínicos bien definidos. Las características genéticas del ADNmt, herencia materna, poliplasmia y segregación mitótica, confieren a estas enfermedades propiedades muy particulares. Las manifestaciones clínicas de estas patologías son muy heterogéneas y afectan a distintos órganos y tejidos por lo que su correcto diagnóstico implica la obtención de datos clínicos, morfológicos, bioquímicos y genéticos.

Por otra parte, la investigación de las diferencias genéticas subyacentes, heredadas por individuos predispuestos a la producción de células TH2, en respuesta a alérgenos alimentarios, promete identificar mecanismos involucrados en determinar el desarrollo de la tolerancia inmunológica o en su defecto la enfermedad alérgica (20). En este sentido, la variabilidad clínica observada en la presentación de la ALV, podría explicarse en parte, por la presencia de polimorfismos en genes candidatos que influyen en su desarrollo.

Las variaciones nucleotídicas encontradas en el ADNmt humano determinan los haplogrupos, los cuales trazan la ascendencia matrilineal hasta los orígenes de la especie humana en África y desde allí a su dispersión geográfica global. Los haplogrupos más

Revista Methodo: Investigación Aplicada a las Ciencias Biológicas. Facultad de Medicina. Universidad Católica de Córdoba. Jacinto Ríos 571 Bo Gral. Paz. X5004FXS. Córdoba. Argentina. Tel.: (54) 351 
Boudet RV, Copioli JC, Muiño JC, Geréz de Burgos N, Chaig MR, Damilano G, Chaig R. Identificación de población de riesgo para alergia a la leche de vaca

antiguos son más grandes y se encuentran más dispersos y de ellos descienden numerosos subgrupos. La migración, dando por resultado el aislamiento de la población, es una de las cuatro fuerzas evolutivas junto a la selección natural, deriva genética y mutación. La disciplina de genética de la población (Gp), es el estudio de la distribución de frecuencias y del cambio en la variación del gen (alelo) bajo tales influencias (21).

La Gp observada en poblaciones modernas, ha abierto una ventana en los patrones históricos de migraciones, con una técnica iniciada por Luigi Luca Cavalli-Sforza (22).

Nuestro estudio, se concentró en conocer además de los haplogrupos nativos en América, qué otros haplogrupos en el ADNmt se presentan en la población estudiada (mediados por la inmigración y el medio ambiente $u$ otros factores) y de qué manera influyen en el desarrollo de la enfermedad ALV. Para observar las posibles relaciones genealógicas entre los distintos compases, utilizamos una técnica común en el análisis filogenético que nos ayudara a analizar y visualizar el conjunto de datos obtenidos en el cuadro (matriz) de distancias. Esta técnica de análisis de datos se basa en la generación de los llamados árboles filogenéticos, que son estructuras geométricas de interconexión que representan la posible relación entre las distintas especies en estudio. El común ancestro más reciente es el ADNmt, con quién se alinearon cada uno de los niños incluidos en este estudio.

El interrogatorio realizado a sus padres, denunció un origen ancestral como descendientes de españoles, italianos y criollos. Sin embargo, la presencia del haplogrupo $Q$ que deriva del $M$ y éste del L3 (Africano), en este grupo de niños, indicaría migración que da origen a la diversidad del mestizaje $(22,23)$.

Por otra parte, no es frecuente que los niños ALV comiencen la enfermedad con la afectación del sistema respiratorio, como única manifestación clínica (8). Al respecto, la variante no descripta (cambio nucleotídico T16519C) estuvo presente solo en los niños ALV que iniciaron la enfermedad con manifestaciones clínicas gastrointestinales y dérmicas.

\section{CONCLUSIONES}

La variante no descripta (T16519C) se presentó con mayor frecuencia en los niños que habían sido diagnosticados como ALV.

El riesgo de desarrollar ALV fue 3 veces más elevado en los pacientes que la poseían.

Todos los niños ALV que iniciaron la enfermedad con manifestaciones clínicas de Dermatitis Atópica (DA) y Enfermedad Gastrointestinal (EGI), la 
Boudet RV, Copioli JC, Muiño JC, Geréz de Burgos N, Chaig MR, Damilano G, Chaig R. Identificación de población de riesgo para alergia a la leche de vaca

presentaron. Esto sugiere que la

presencia de esta mutación

probablemente aumente la posibilidad

de padecer ALV asociada con DA+EGI.

En el desarrollo de la ALV estarían

involucrados además del ADN

mitocondrial otros genes nucleares y

factores epigenéticos que hacen al

fenoma y quizás al proteoma. Futuras

\section{BIBLIOGRAFÍA}

1. Leung PSC, Shu S-A, Chang C. Clinic Rev Allerg Immunol 2014; 46:169-179

2. Tan N TH, Ellis JA, Saffery R, Allen $\mathrm{KJ}$. The role of genetics and environment in the rise of childhood food allergy. Clin Exp Allergy 2012; 42:20-29.

3. Rona RJ, Keil T, Summers C, Gislason D, Zuidmeer L, Sodergren $E$, et al. The prevalence of food allergy: a meta-analysis. J Allergy Clin Immunol 2007; 120:638-46.

4. Hourihane JO, Dean TP, Warner JO. Peanut allergy in relation to heredity, maternal diet, and other atopic diseases: results of a questionnaire survey, skin prick testing, and food challenges. BMJ1996; 313:518-21.

5. Tsai HJ, Kumar R, Pongracic J. Familial aggregation of food allergy and sensitization to food allergens: a family-based study. Clin Exp Allergy 2009; 39:101-9.

6. Chinthrajah RS, Hernandez JD, Boyd SD, Galli SJ, Nadeau KC. Molecular and cellular mechanisms of food allergy and food tolerance. J Allergy Clin Immunol. 2016 ;137(4):984-97.

7. Ortolani C, Pastorello EA. Food allergies and food intolerances. Best Pract Res Clin Gastroenterol 2006; 20:467-83.

8. Fiocchi A, Brozek J, Schunemann H, Bahna SL, Von Berg A, Beyer K, et al. World Allergy Organization (WAO) Diagnosis and Rationale for Action against Cow's Milk Allergy (DRACMA) Guidelines. Pediatr Allergy Immunol 2010; 21:1-125. investigaciones que involucren al $\mathrm{Gmt}$ completo, serán necesarias para poder correlacionar las variantes de la Región Control D-Loop con el o los haplogrupos correspondientes, lo que resultaría en los haplotipos o subhaplotipos que las caracterizan, tanto para la variante no descripta T16519C como para otras hipotéticas.

9. American Academy of Pediatrics Committee on Nutrition. Hypoallergenic infant formulae. Pediatrics 2000; 106:346-49.

10. Muraro A, Werfel T, HoffmannSommergruber K, Roberts $\mathrm{G}$ et al. EAACI Food Allergy and Anaphylaxis Guidelines Group. EAACI Food Allergy and Anaphylaxis Guidelines. Diagnosis and management of food allergy. Allergy 2014; 69: 10081025.

11. Wallace DC, Brown MD, Lott MT. Mitochondrial DNA variation in human evolution and disease. Gene 1999; 238:211-30.

12. Torroni A, Wallace DC. Mitochondrial DNA variation in human populations and implications for detection of mitochondrial DNA mutations of pathological significance. $J$ Bioenerg Biomembr 1994; 26:26171.

13. Brown MD, Torroni A, Reckord CL, Wallace DC. Phylogenetic analysis of Leber's hereditary optic neuropathy mitochondrial DNA's indicates multiple independent occurrences of the common mutations. Hum Mutat 1995; 6:311 25.

14. Kofler B, Mueller EE, Eder W, Stanger $O$, Maier $R$, Weger $M$, Hass A, et al. Mitochondrial DNA haplogroup $T$ is associated with coronary artery disease and diabetic retinopathy: a case control study. BMC Med Genet 2009; 10:35-42. 
Boudet RV, Copioli JC, Muiño JC, Geréz de Burgos N, Chaig MR, Damilano G, Chaig R. Identificación de población de riesgo para alergia a la leche de vaca

15. Wakeley J. Substitution rate variation among sites in hypervariable region 1 of human mitochondrial DNA. J Mol Evol 1993; 37:613-23.

16. Saitou N, Nei M. The Neighborjoining Method: A New Method for Reconstructing Phylogenetic Trees. Mol. Biol. Evol. 1987; 4(4):406-425.

17. Eddy SR. Where did the BLOSUM62 alignment score matrix come from? Nat Biotechnol. 2004; 22(8):1035-6.

18. Jukes $\mathrm{TH}$ and Cantor $\mathrm{CR}$ (1969). Evolution of Protein Molecules. New York: Academic Press. pp. 21-132.

19. Kimura M (1980). "A simple method for estimating evolutionary rates of base substitutions through comparative studies of nucleotide sequences". Journal of Molecular Evolution 16 (2): 111-120.

20. Bedoret D, Singh AK, Shaw V, Hoyte EG, Hamilton R, DeKruyff $\mathrm{RH}$, et al. Changes in antigen- specific $T$ cell number and function during oral desensitization in cow's milk allergy enabled with omalizumab. Mucosal Immunol. 2012; 5: 267-76.

21. Rieder MJ, Tayler SL, Tobe VO, Nickerson DA. Automating the identification of DNA variations using quality-based fluorescence re-sequensing: Analysis of the human mitochondrial genome. Nucleic Acids Res. 1998; 26: 967 73.

22. Piazza A, Rendine S, Minch E, Menozzi P, Mountain J, CavalliSforza LL. Genetics and the origin of European languages. Proc. Natl. Acad. Sci. USA 1995; 92:5836-40.

23. Fagundes N, Kanitz R, Eckert R. Mitochondrial population genomics supports a single pre-Clovis origin with a coastal route for the peopling of the Americas. Book: Biomolecular Archaeology: An Introduction 2008; 284-86. 